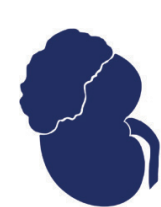

jkcvhl.com

KIDNEY CANCER: CASE REPORT

\title{
Durable Remission with Immunotherapy in a Patient with Sarcomatoid Renal Cell Carcinoma
}

\author{
Nwabundo Anusim, Damilola Gbadebo, Olabisi Afolayan-Oloye, Ishmael Jaiyesimi
}

Department of Hematology and Oncology, Beaumont Health, Royal Oak, MI, USA

\begin{abstract}
Sarcomatoid differentiation is a rare and aggressive histologic subtype with poor prognosis, seen in several malignancies. In sarcomatoid renal cell carcinoma (RCC), the degree of sarcomatoid differentiation and the stage at presentation determines the prognosis. Despite resection, chemotherapy and targeted therapy response is modest, with relapse usually occurring within a few months. We present a case of a gentleman with sarcomatoid RCC managed with pembrolizumab, who has had no evidence of recurrence for over 4 years since the last dose of immunotherapy. RCCs with sarcomatoid differentiation have a high presence of programmed cell death protein 1 and programmed cell death ligand 1 in T cells and tumor cells, respectively, making immunotherapy an attractive option in this setting. Clinical trials are ongoing to further define the benefit of immunotherapy in sarcomatoid RCC.
\end{abstract}

Keywords: case study; immunotherapy; renal cell carcinoma; sarcomatoid differentiation

Received: 16 January 2021; Accepted after revision: 11 August 2021; Published: 26 October 2021

Author for correspondence: Nwabundo Anusim, MD, Department of Hematology and Oncology, Beaumont Health, 3601 W. 13 Mile Rd, Royal Oak, USA. Email: nwabundo.anusim@austin.utexas.edu

How to cite: Anusim N, et al. Durable Remission with Immunotherapy in a Patient with Sarcomatoid Renal Cell Carcinoma. J Kidney Cancer VHL. 2021; 8(4): 38-42.

Doi: https://doi.org/10.15586/jkcvhl.v8i4.168

Copyright: Anusim N, et al.

License: This open access article is licensed under Creative Commons Attribution 4.0 International (CC BY 4.0). http://creativecommons.org/ licenses/by/4.0

\section{Case Presentation}

A 73-year-old man presented to our facility with complaints of left upper quadrant pain and twenty-pound weight loss. A computed tomographic (CT) scan of the chest, abdomen, and pelvis revealed a huge upper quadrant complex mass (Figure 1A), whereas a biopsy of the mass revealed high-grade malignant neoplasm. He underwent radical nephrectomy, splenectomy, and resection of the diaphragm. Pathology revealed a $23.5 \mathrm{~cm}$ mass with histologic grade 4 renal cell carcinoma (RCC) with sarcomatoid (95\%) and focal papillary $(1 \%)$ features that invaded into the perinephric fat, gerota fascia, spleen, and diaphragm, all with negative margins, translating to a pathological stage 4 . On immunohistochemistry, the tumor stained positive for CK 7, PAX-8, and CD-10, whereas AE1/AE3 was focally positive (Figure 1B-E). Three months postnephrectomy, he complained of pleuritic chest pain. A CT scan of the chest revealed abnormal tissue in the inferior posterior left pleural 
(A)

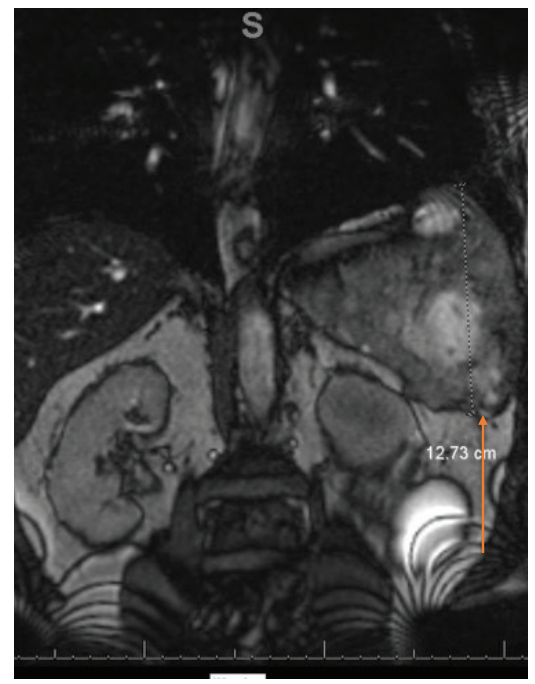

(B)

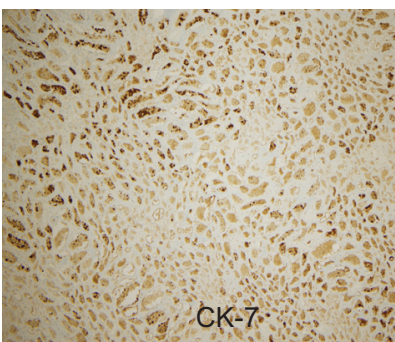

(D)

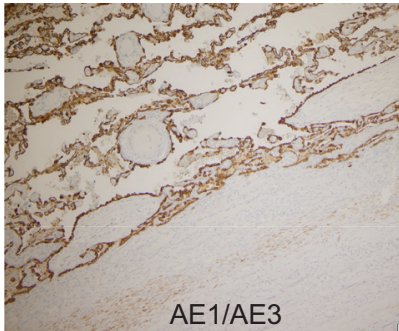

(C)

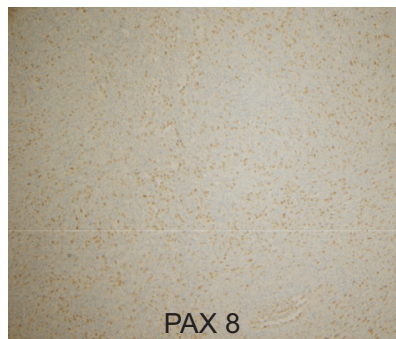

$(\mathrm{E})$

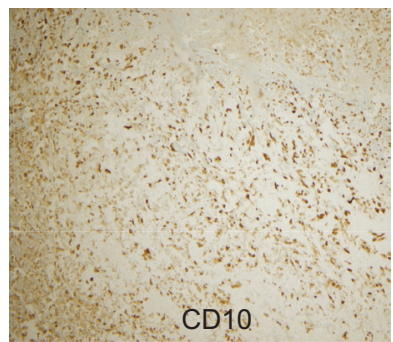

(F)

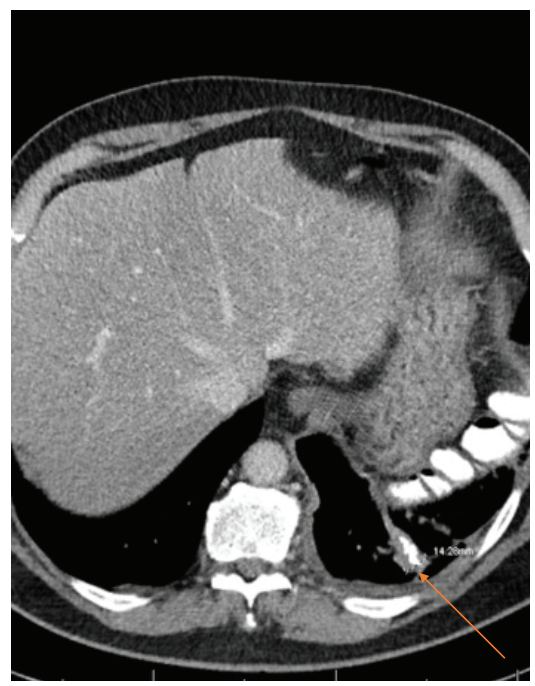

(G)

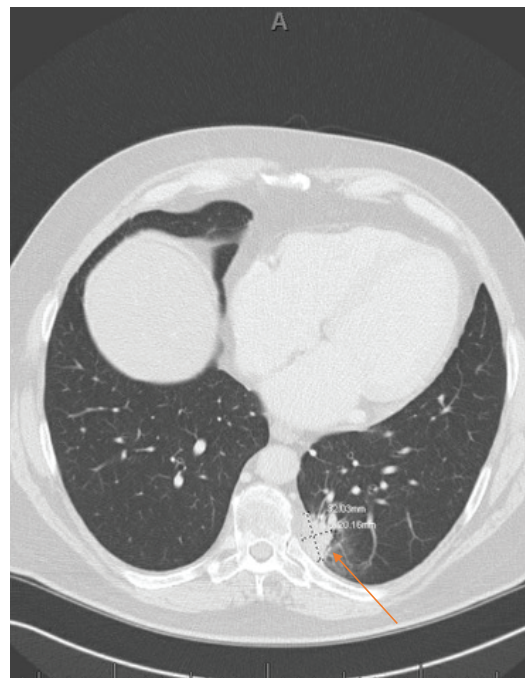

Figure 1: Radiologic and pathologic findings.

space, and left-sided abdominal and peritoneal lymph nodes. Biopsy revealed sarcomatoid $\mathrm{RCC}$. He received radiation therapy to that region and subsequent chemotherapy with sunitinib and gemcitabine. A repeat CT of the chest, abdomen, and pelvis 6 months after therapy initiation revealed no evidence of disease.

Unfortunately, 9 months after chemotherapy initiation, the patient noted a tender subcutaneous nodule in the left chest wall. CT scan of the chest revealed a new irregular area of pleural soft tissue at the posterior medial aspect of the left lower lobe of the lung (Figure 1G). A positron emission tomography (PET) scan revealed multiple suspicious retrocrural and left aortic lymph nodes with intense 18F-fluorodeoxyglucose (FDG) avidity, in addition to several FDGavid soft tissue lesions in the left chest and abdominal wall. He underwent radiation to the left abdominal wall and due to his high PD-1 and PD-L1 status he was prescribed pembrolizumab, which he received for only 3 months because of grade 4 immune-mediated pneumonitis. Repeat PET scans showed interval resolution of the previous lesions in the peritoneal and retroperitoneal cavities and the posterior left costophrenic angle, indicating a favorable response to therapy. Recent PET scans after more than 4 years of immunotherapy revealed no evidence of recurrent disease. 


\section{Discussion}

Sarcomatoid RCC is a rare histologic subtype, present in $5 \%-8 \%$ of all RCCs (1). It is aggressive with a poor prognosis and overall survival of approximately 4 months when diagnosed at an advanced stage (2). Sarcomatoid RCC can also be seen in combination with other subtypes of RCC, where a higher percentage of sarcomatoid features confers a worse prognosis (3). Surgical resection is curative in early-stage disease. However, in advanced disease, there is typically a rapid onset of relapse despite complete surgical resection. In a retrospective review of patients with metastatic chromophobe RCC, patients with sarcomatoid features versus those without had a statistically significant shorter median time to metastatic disease relapse after nephrectomy for localized disease (2.7 months vs. 48.8 months) and treatment failure during first-line treatment (1.8 months vs. 8 months) when compared with those without sarcomatoid features (4). Chemotherapeutic agents have limited success in producing response or durable remission. In a phase 2 trial of doxorubicin and gemcitabine in 39 patients with sarcomatoid RCC, $16 \%$ of patients experienced response, whereas $26 \%$ had stable disease. The median progression-free survival (PFS) was 3.5 months, and the median overall survival (OS) was 8.8 months (5). Tyrosine kinase inhibitors, mammalian target of rapamycin (mTOR) inhibitors, and vascular endothelial growth factor inhibitors with chemotherapy have been utilized with modest improvement in survival $(6,7)$. In a phase 2 trial of capecitabine, gemcitabine, and bevacizumab in patients with metastatic or unresectable sarcomatoid RCC, the response rate remained low, with only $20 \%$ of the participants achieving a response and only one patient with a complete response. The median PFS in this trial was 5.5 months, and the median OS was 12 months. About $91 \%$ of patients discontinued treatment because of treatment-related toxicity, commonly noted with combination therapies (7). In a retrospective study of patients with sarcomatoid RCC who received sunitinib, PFS was 5.7 months, with only $30 \%$ of patients achieving stable disease (1). In another retrospective study assessing the benefit of mTOR inhibitors in 23 patients with sarcomatoid RCC, the median PFS and OS were 3.5 months and 8.2 months, respectively (6).

RCCs with sarcomatoid differentiation have been noted to have $89 \%$ programmed cell death ligand 1 (PDL-1) positivity and combined PDL-1 and programmed cell death protein-1 positivity of $50 \%$ (8). Despite this, studies have shown conflicting responses to immunotherapy. In a retrospective review of patients with sarcomatoid RCC treated with immunotherapy versus chemotherapy, a triple improvement in the median PFS in the immunotherapy group and no difference in OS was observed (9). In a single case report, a notably durable remission of about 2 years was seen in a patient who received nivolumab (10). Our patient achieved an ongoing 4-year remission after treatment with pembrolizumab.
The phase 3 Checkmate 426 trial comparing the combined targeted agents axitinib and pembrolizumab versus sunitinib in 105 patients with sarcomatoid RCC showed impressive results in favor of combined axitinib and pembrolizumab. There was an almost doubling of the objective response rate (ORR, $58.8 \%$ vs. $31.5 \%$ ) and a complete response rate (CRR) of $11.8 \%$ vs. $0 \%$. The 12 -month overall OS showed a $42 \%$ reduction in the risk for death in favor of pembrolizumab and axitinib (83.4\% vs. 79.5\%) (11).

In the recent phase 3 Checkmate 214 trial, which compared dual immunotherapy (nivolumab and ipilimumab) with sunitinib, in patients with advanced RCC with sarcomatoid features the median OS was not reached at a minimum follow-up of 42 months in the immunotherapy arm compared with 14.2 months in the sunitinib arm, which translated to a statistically significant $55 \%$ reduction in the risk for death with dual immunotherapy. In addition to the impressive OS benefit, the ORR and CRR were 2.6 and 6.1 times higher, respectively, than with sunitinib (60.8\% vs. $23.1 \%$ and $18.9 \%$ vs. $3.1 \%)(12)$.

Considering these impressive data, an immune checkpoint-based regimen should be the initial choice in the management of patients with metastatic or advanced sarcomatoid RCC over local therapies like nephrectomy and radiation therapy.

Patients are encouraged to participate in clinical trials for further treatment developments with improved efficacy and limited toxicities. Currently, clinical trials are in progress using immunotherapy and immunotherapy in combination with targeted agents to define the benefits of immunotherapy in sarcomatoid RCC. However, patient accrual in these trials may be difficult because of the rarity of this histologic subtype. Umbrella trials are an effective means of investigating different cancers with sarcomatoid histology to assess biomarkers and treatment options (Table 1).

\section{Ethics Approval}

This study was determined to be exempt from IRB review by the Beaumont Health Human Investigation Committee.

\section{Conflict of Interests}

The authors declare no competing interests.

\section{Funding}

This study received no funding from the public, private, or not-for-profit sectors.

\section{Authors' Contributions}

All authors contributed equally to the conception, data collection, and manuscript preparation for this study. 


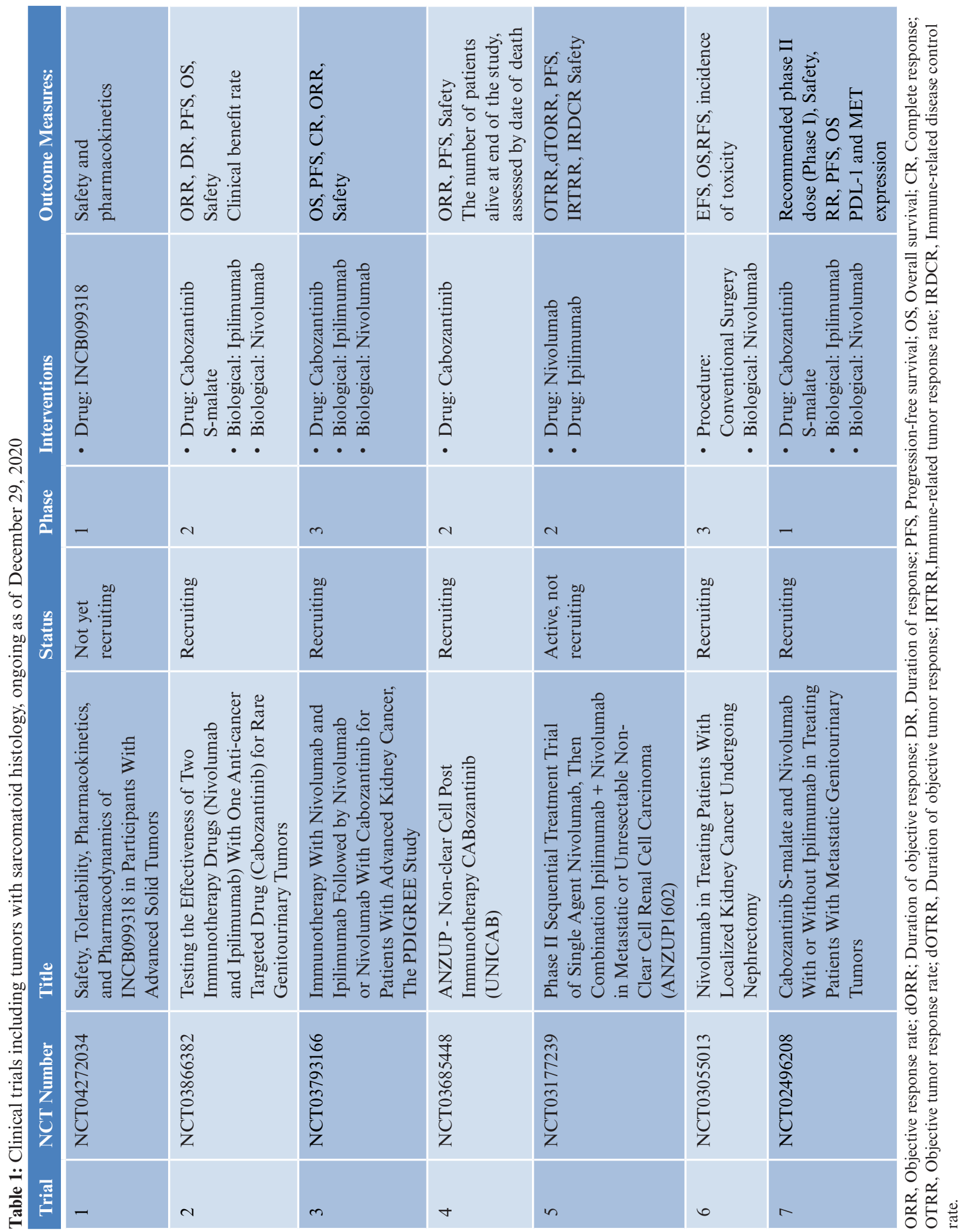




\section{References}

1. Kunene V, Miscoria M, Pirrie S, Islam MR, Afshar M, Porfiri E. Sarcomatoid renal cell carcinoma: Clinical outcome and survival after treatment with sunitinib. Clin Genitourin Cancer. 2014;12(4):251-255. http://dx.doi.org/10.1016/j.clgc.2013.12.001

2. Keskin SK, Msaouel P, Hess KR, Yu KJ, Matin SF, Sircar K, et al. Outcomes of patients with renal cell carcinoma and sarcomatoid dedifferentiation treated with nephrectomy and systemic therapies: Comparison between the cytokine and targeted therapy eras. J Urol. 2017;198(3):530-537. http://dx.doi. org/10.1016/j.juro.2017.04.067

3. Shuch B, Bratslavsky G, Shih J, Vourganti S, Finley D, Castor B, et al. Impact of pathological tumour characteristics in patients with sarcomatoid renal cell carcinoma. BJU In. 2012;109(11):1600-1606. http://dx.doi. org/10.1111/j.1464-410X.2011.10785.X

4. Ged Y, Chen YB, Knezevic A, Casuscelli J, Redzematovic A, DiNatale RG, et al. Metastatic chromophobe renal cell carcinoma: Presence or absence of sarcomatoid differentiation determines clinical course and treatment outcomes. Clin Genitourin Cancer. 2019 Jun;17(3):e678-e688. http://dx.doi.org/10.1016/j. clgc.2019.03.018

5. Haas NB, Lin X, Manola J, Pins M, Liu G, McDermott D, et al. A phase II trial of doxorubicin and gemcitabine in renal cell carcinoma with sarcomatoid features: ECOG 8802. Med Oncol. 2012;29(2):761-767. http://dx.doi.org/10.1007/ s12032-011-9829-8

6. Voss MH, Bastos DA, Karlo CA, Ajeti A, Hakimi AA, Feldman DR, et al. Treatment outcome with mTOR inhibitors for metastatic renal cell carcinoma with nonclear and sarcomatoid histologies. Ann Oncol. 2014;25(3):663-668. http:// dx.doi.org/10.1093/annonc/mdt578

7. Maiti A, Nemati-Shafaee M, Msaouel P, Pagliaro LC, Jonasch E, Tannir NM, et al. Phase 2 trial of capecitabine, gemcitabine, and bevacizumab in sarcomatoid renal-cell carcinoma. 2017; Aug:S1558-7673(17)30238-0. http://dx.doi.org/10.1016/j. clgc.2017.07.028

8. Joseph RW, Millis SZ, Carballido EM, Bryant D, Gatalica Z, Reddy S, et al. PD-1 and PD-L1 expression in renal cell carcinoma with sarcomatoid differentiation. Cancer Immunol Res. 2015;3(12):1303-1307. http://dx.doi.org/10.1158/2326-6066. CIR-15-0150

9. Kwak C, Park YH, Jeong CW, Jeong H, Lee SE, Moon KC, et al. Sarcomatoid differentiation as a prognostic factor for immunotherapy in metastatic renal cell carcinoma. J Surg Oncol. 2007;95(4):317-323. http://dx.doi.org/10.1002/jso.20669

10. El Mouallem N, Smith SC, Paul AK. Complete response of a patient with metastatic sarcomatoid renal cell carcinoma to a programmed death-1 checkpoint inhibitor. J Oncol Pract. 2018;14(8):511-513. http://dx.doi.org/10.1200/JOP.18.00213

11. Rini BI, Plimack ER, Stus V, Gafanov R, Hawkins R, Nosov D, et al. Pembrolizumab plus Axitinib versus Sunitinib for Advanced Renal-Cell Carcinoma. N Engl J Med. 2019 Mar; 380(12):1116-1127. http://dx.doi.org/10.1056/NEJMoa1816714

12. Tannir NM, Signoretti S, Choueiri TK, McDermott DF, Motzer RJ, Flaifel A, et al. Efficacy and safety of nivolumab plus ipilimumab versus sunitinib in first-line treatment of patients with advanced sarcomatoid renal cell carcinoma. Clin Cancer Res. 2021Jan;27(1):78-86. http://dx.doi.org/10.1158/1078-0432.CCR20-2063 